\title{
Modelos de planeamiento energético aplicados en Perú: una revisión y propuesta metodológica
}

\author{
José Neil Meza Segura', Jaime Luyo Kuong² \\ ${ }^{1}$ Programa de Doctorado en Ciencias con Mención en Energética, Universidad Nacional de Ingeniería, Av. \\ Tupac Amaru 210, Rímac, Lima, Perú \\ ${ }^{2}$ Facultad de Ingeniería Mecánica, Universidad Nacional de Ingeniería, Av. Tupac Amaru 210, Rímac, Lima, \\ Perú
}

Presentado el 31 de diciembre 2020. Revisado 13 de enero. Aprobado 13 de febrero 2020

DOI: https://doi.org/10.33017/RevECIPeru2020.0002/

\section{Resumen}

Un modelo energético sirve de base para realizar estudios de prospectiva. Sin embargo, en el contexto internacional de lucha contra el cambio climático y negociaciones internacionales de reducción de GEI, se plantean nuevos retos y paradigmas que los enfoques metodológicos deben cumplir. En el presente artículo se realiza una evaluación de los modelos energéticos empleados en estudios de planeamiento del sistema energético peruano, clasificándolos y evaluando el cumplimiento de paradigmas que plantean los sistemas energéticos actuales. Finalmente, para cumplir con los nuevos retos y paradigmas, se plantea una propuesta metodológica hibrida que cuenta con cuatro componentes: de uso final, de optimización, de integración del balance energético y de evaluación de escenarios simulados.

Descriptores: modelos energéticos, matriz energética, prospectiva energética, balance de energía, gases de efecto invernadero (GEI)

\section{Abstract}

An energy model serves as the basis for prospective studies. However, in the international context of combating climate change and international GHG reduction negotiations, it poses new challenges of paradigms that methodological approaches must meet. In this article an evaluation of the energy models used in planning studies of the Peruvian energy system is carried out, classifying them and evaluating the fulfillment of paradigms posed by current energy systems. Finally, to meet the new challenges and paradigms, a hybrid methodological proposal is proposed that has four components: end use, optimization, integration of the energy balance and evaluation of simulated scenarios.

Keywords: energy models, energy matrix, energy prospective, energy balance, greenhouse gases (GHG)

\section{Introducción}

Un modelo energético sistematiza y facilita la representación del funcionamiento de la matriz energética de un país, esto con la finalidad de evaluar prospectivas y formular políticas; por lo que, para su elaboración deben de considerarse una serie de etapas que ayuden a dimensionar los alcances del planteamiento metodológico que se desea implementar [1].
Como Pfenninger et al [2] manifiesta, existen retos de los sistemas energéticos del siglo XXI, y se debe tener cuidado al seleccionar el modelo energético o metodología más adecuada para hacer frente a un problema específico que se quiera analizar, debido a que a veces se hace uso de modelos ya establecidos gracias a la familiaridad en su uso; a fin de superar dichas limitaciones, se debe innovar con métodos híbridos y/o provenientes de otros campos del conocimiento, asegurándose de que los efectos del incremento de la complejidad puedan 
ser capturados adecuadamente, para lo cual una fuente de mejora importante es la aplicación de los estándares de trabajo desarrollados dentro de la comunidad de desarrollo de software de código abierto.

El contexto internacional actual donde la lucha contra el cambio climático a través de la mitigación GEI aparentemente se había consolidado en el acuerdo de París de la COP21; sin embargo, luego de análisis realizados se demostró que las contribuciones previstas y determinadas a nivel nacional (iNDC por sus siglas en inglés) propuestas por las naciones serán insuficientes para llegar a la meta de mantener el incremento de temperatura por debajo de los $2^{\circ} \mathrm{C}$ [3] y [4],

Asimismo, si se tiene en cuenta que la región de Latino América tiene características marcadamente diferentes con el resto de países, como es el alto componente y potencial de recursos renovables, y precisamente a través de dichos recursos es la electricidad, el energético que tiene la mayor potencialidad de ser una opción económicamente viable para la descarbonización de otros sectores; sin embargo, la mayor fuente de emisiones en la región es la deforestación y cambio uso de suelos [5] que es donde se concentran el porcentaje más alto de reducciones de GEl asumido por el Perú a través de medidas de mitigación que serán de difícil cumplimiento debido a la envergadura de la Amazonía [6]; Debido a esto, se hace de gran necesidad que el planeamiento energético, incluya un análisis estratégico y evalué las implicancias que conllevan los compromisos asumidos en estos campos en especial en los países en vías de desarrollo como el Perú; así como, si dichos compromisos son realizables y si existe la potencialidad de ampliar las metas de reducciones; así como, evaluar si dichos compromisos están en concordancia con la política energética del país [7].

Si bien es cierto el Perú tiene experiencia en la aplicación periódica de modelos para el planeamiento energético, esto se realizan de forma sectorial y aislada, por ejemplo, en el sector de electricidad se realizan estudios para la fijación de tarifas en barra [8], planeamiento de la transmisión [9], planes referenciales de electricidad [10], planes de inversión de subtransmisión [11], planes de inversiones en distribución [12], plan nacional de electrificación rural [13]; mientras que en el sector hidrocarburos se tiene el plan de desarrollo de los recursos de hidrocarburos [14] y el plan quinquenal de inversiones en distribución de gas natural [15]. Sin embargo, la experiencia en el uso de modelos integrales del sector energético es mucho más limitada.

En vista de esto, el desafío para el Perú se sintetiza en mejorar su sistema de planificación energética de manera que se identifique una metodología para la planificación estratégica energética de largo plazo que posibilite el desarrollo energético sostenible del país, y pueda cumplir con la política energética del país e identifique los diferentes componentes que ayudaran en la lucha contra el cambio climático tanto en el lado de la oferta como en la demanda de energía; asimismo, este dimensionado para países en vías de desarrollo, ya que muchas veces los resultados o atributos que se pretenden conseguir son claramente contrapuestos; por ejemplo, se busca suministrar energía barata pero que genere la menor cantidad de emisiones de GEI posibles.

El presente artículo hace una revisión de los modelos energéticos integrales que fueron empleado por diversos estudios $\mathrm{y} / \mathrm{o}$ planes energéticos en el Perú. Establece criterios para su clasificación y evaluación a fin de identificar falencias y oportunidades de mejora, para finalmente hacer una propuesta metodológica que pueda ser estandarizada, actualizada periódicamente y utilizada por diferentes entes del sector energía; así como, por la academia como base para estudios de prospectiva energética.

\section{Metodología}

Los modelos energéticos seleccionados para el análisis en el presente artículo son aquellos que hayan sido empleados en el marco de un estudio de prospectiva para la totalidad del sistema energético peruano, es decir no se tomaron en cuenta los planes sectoriales. Dichos estudios fueron elaborados tanto por instituciones gubernamentales como no gubernamentales, peruanas y extranjeras.

Una vez identificados los estudios a evaluar, se hace una evaluación de la información publicada disponible y se presenta una breve descripción de cada uno, posteriormente se categorizan de acuerdo con el tipo de modelos o metodologías empleados, para ello se emplea una variante de las categorizaciones propuestas en Van Beeck [16], Urban et al [17] y Hall et al [18].

Seguidamente, dada que dichos ejercicios de planificación servirán de base para la formulación de políticas, y futuros compromisos internacionales de reducción de emisiones, debe proporcionar respuestas adecuadas a los desafíos actuales y futuros, se evalúa si dichos modelos cumplen con 
los siguientes paradigmas identificados por Huppmann et al en [19] y otros identificados en el presente artículo:

i) Objetivos de políticas cuantificados

ii) Evaluación de escenarios modelados

iii) Adaptarse a complejidad creciente

iv) Interacción con otros sectores

v) Transparencia, reproductibilidad e inteligibilidad

Finalmente, se presenta una propuesta de enfoque metodológico que cumpla con dichos paradigmas.

\section{Resultados}

\subsection{Estudios Analizados}

La Tabla 1, presenta los estudios y/o planes elaborados en los últimos años en el Perú y el grado de información disponible, siendo el más antiguo y por ende con menor información disponible el EIEP; mientras que tanto el SPEN y el APEC son los estudios de reciente publicación y el PLANCC el que ofrece más información disponible a consultar.

Tabla 1: Estudios Analizados e Información Disponible

\begin{tabular}{|l|c|c|c|}
\hline \multicolumn{1}{|c|}{ Acrónimos y Nombre del Estudio } & $\begin{array}{c}\text { Informe del } \\
\text { Estudio }\end{array}$ & $\begin{array}{c}\text { Documentación } \\
\text { del Modelo }\end{array}$ & $\begin{array}{c}\text { Datos } \\
\text { Empleados }\end{array}$ \\
\hline EIEP (Estudio Integral Energético del Perú) & $\mathrm{NO}$ & $\mathrm{NO}$ & $\mathrm{NO}$ \\
\hline $\begin{array}{l}\text { NUMES (Nueva Matriz Energética Sostenible y } \\
\text { Evaluación Ambiental Estratégica, como } \\
\text { Instrumentos de Planificación) }\end{array}$ & $\mathrm{SI}$ & $\mathrm{SI}$ & $\mathrm{NO}$ \\
\hline PEN (Plan Energético Nacional 2014 - 2025) & $\mathrm{SI}$ & $\mathrm{NO}$ & $\mathrm{SI}$ \\
\hline PLANCC (Planificación Ante el Cambio Climático) & $\mathrm{SI}$ & $\mathrm{SI}$ & $\mathrm{SI}$ \\
\hline $\begin{array}{l}\text { SPEN (Sistema de Planificación Energética } \\
\text { Nacional) }\end{array}$ & $\mathrm{SI}$ & $\mathrm{SI}$ & $\mathrm{NO}$ \\
\hline $\begin{array}{l}\text { APEC (Asia-Pacific Economic Cooperation Energy } \\
\text { Outlook 2019) }\end{array}$ & $\mathrm{SI}$ & $\mathrm{SI}$ \\
\hline
\end{tabular}

A. Estudio Integral Energético de Perú [20], realizado el año 2001 a solicitud de la oficina técnica de energía del MINEM, empleó el modelo LEAP (versión 2000); sin embargo, debido a la antigüedad y poca socialización que tuvo el estudio no se cuenta con información necesaria que pueda ser analizada a detalle.

B. Nueva Matriz Energética Sostenible y Evaluación Ambiental Estratégica, como Instrumentos de Planificación [21], realizada el año 2012 a solicitud de la DGEE del MINEM. Sobre dicho estudio Luyo [22] concluyó que el estudio representó "una primera aproximación al desarrollo del planeamiento energético nacional de largo plazo"; sin embargo, también indicó que el planteamiento metodológico manifestó limitaciones.

C. Plan Energético Nacional 2014 - 2025 [23] elaborada el año 2014 por la DGEE del MINEM. Respecto a dicho estudio Ríos [24] manifestó que las proyecciones de demanda de largo plazo fueron basadas en un modelo económico de crecimiento infinito y sugirió incluir planes de ahorro y eficiencia energética dentro de dichas proyecciones.
D. Planificación Ante el Cambio Climático PLANCC [25], fue elaborado, a solicitud del MINAM en coordinación con el MEF, RREE y CEPLAN, en el marco del programa MAPS [26], dicho estudio tuvo por finalidad generar las bases científicas y políticas para explorar la factibilidad de una economía baja en carbono e incorporar el enfoque de cambio climático en la planificación del desarrollo del país; asimismo busco el desarrollo de las capacidades locales en temas de planificación. PLANCC involucró la participación de diversas entidades públicas, privadas y no gubernamentales [27]. Al tener un enfoque macro creo grupos de investigación en los diversos sectores causantes de las emisiones de GEI y como parte del equipo de investigación en el sector energía designó a CENERGIA. Dentro de dicho sector el alcance de la investigación consideró la proyección de la matriz energética, el cálculo de emisiones de GEI hasta el año 2050 y la identificación de opciones de mitigación. Los resultados del estudio sirvieron como base para el desarrollo de la iNDC peruana.

E. Sistema de Planificación Energética Nacional, elaborado el año 2019 a solicitud de la DGEE del 
MINEM con apoyo PROSEMER [28]; dicho estudio incluyó la creación de un sistema de información energética, un sistema de planificación y la elaboración de planes energéticos regionales.

F. APEC Energy Outlook 2019 [29], elaborado por la APERC y el IEEJ para todos los países del Asia - Pacifico, dentro del estudio se realiza la proyección de la matriz energética peruana para los escenarios BAU, TGT y 2DC, para ello aplican la metodología planteada en [30].

En ninguno de los estudios listados, se estandarizó la metodología empleada, tampoco se tuvo acceso a la información primaria que sirvió de base para realizar los análisis. Sin embargo, si comparamos las proyecciones de la demanda en sus diferentes escenarios como se presenta en la Figura 1, se aprecia una marcada diferencia entre los dos escenarios del PEN que resaltan por las elevadas tasas de crecimiento que tendrían llegando la proyección del escenario PEN PBI 6.5 del año 2025 a niveles de demanda proyectados por la NUMES y por SPEN para el año 2040. En el lado contrario se encuentran las proyecciones realizadas por APEC, cuyas tasas de crecimiento son bajas, lo que significaría un escaso crecimiento del sector energético peruano en los próximos años. Otro punto resaltante es la notable concordancia entre la proyección realizada por la NUMES en su escenario BAU y el SPEN ya que se tratan de dos estudios con metodologías marcadamente diferentes como se verá más adelante.

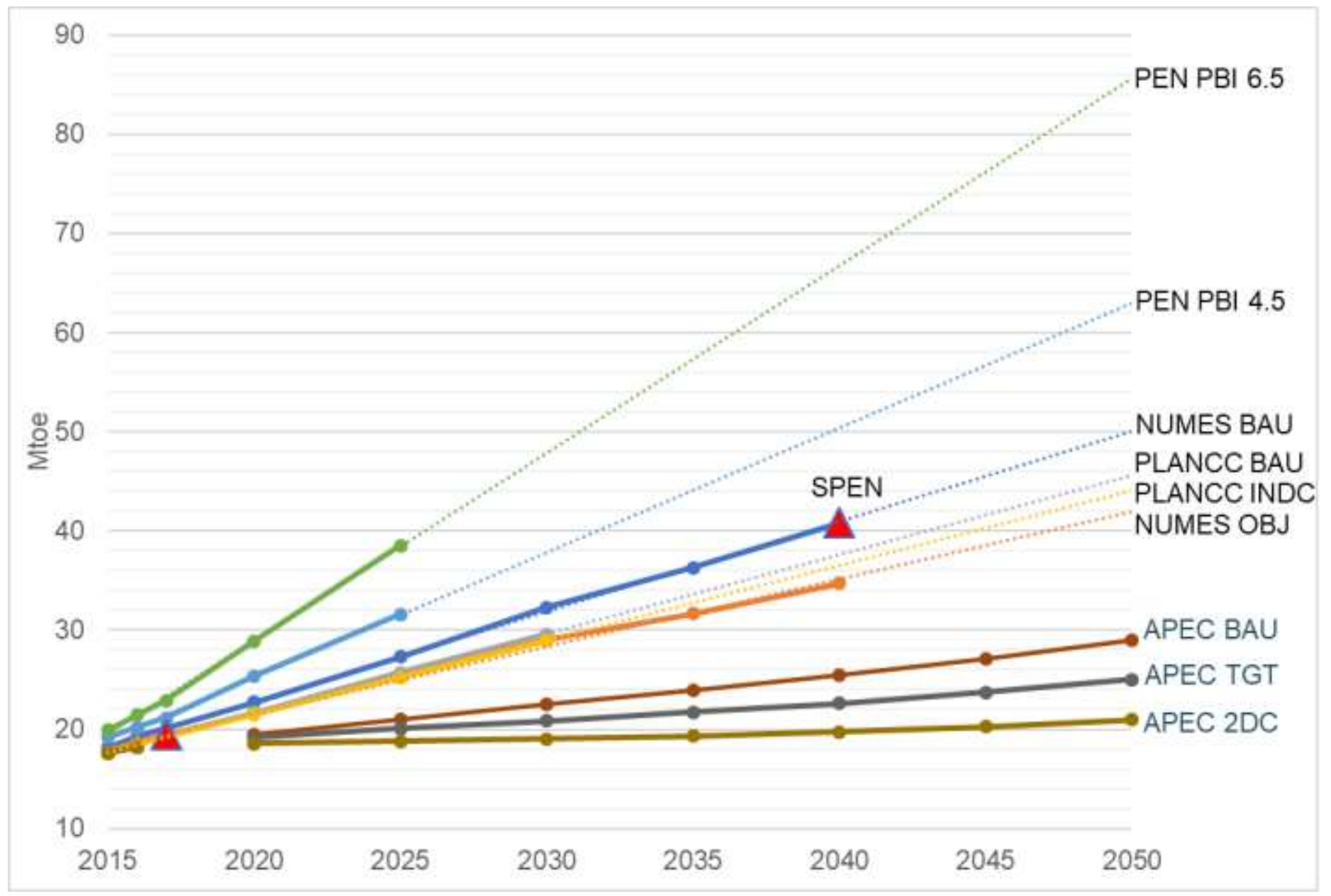

Figura 1: Comparación de las proyecciones de la demanda de los escenarios evaluados en los estudios analizados

\subsection{Clasificación Metodologías}

La tabla 2, presenta la clasificación de las metodologías empleadas en los estudios analizados, se aprecia que en su mayoría los estudios han empleado metodologías híbridas combinando para proyección de la demanda métodos por uso final y métodos econométricos. Asimismo, en el caso de NUMES y PLANCC si bien la oferta de energía empleo métodos de simulación, 
la componente de electricidad si fue optimizada. Los modelos energéticos convencionales empleados son el LEAP y TIMES.

\subsection{Cumplimiento de Paradigmas}

La tabla 3, presenta la evaluación del cumplimiento de los paradigmas identificados, siendo evidente que ninguno de los estudios evaluados serían capaces de adaptarse a la complejidad creciente de los sistemas energéticos ya que dichos estudios son productos de consultorías que ya concluyeron y dejaron los modelos ya establecidos para su uso, ligado a este factor está el hecho de que ninguno cumple con el paradigma de transparencia, reproductividad e inteligibilidad ya que al ser desarrollados en modelos con licencia son de código cerrado y no están disponible para su evaluación. Por otro lado, solo la NUMES realizó una evaluación de los escenarios evaluados a fin de identificar un escenario robusto y tiene la capacidad de interactuar con el sector ambiental a través de su evaluación ambiental estratégica. Mientras que tanto PLANCC como el SPEN tienen la capacidad de interactuar con el sector económico a través de un modelo de equilibrio general computable capaz de identificar el impacto de los escenarios evaluados en la economía nacional.

Tabla 2: Clasificación de las metodologías

\begin{tabular}{|c|c|c|c|c|}
\hline Estudio & Enfoque de la Demanda & Enfoque de la Oferta & $\begin{array}{c}\text { Integración Sistema } \\
\text { Energético }\end{array}$ & $\begin{array}{c}\text { Horizonte } \\
\text { Temporal }\end{array}$ \\
\hline EIEP & Uso Final & Simulación & LEAP (versión 2000) & $2000-2010$ \\
\hline NUMES & $\begin{array}{c}\text { Gas Natural: Uso Final } \\
\text { Resto: Econométrico }\end{array}$ & $\begin{array}{c}\text { Electricidad: Optimización } \\
\text { Resto: Simulación }\end{array}$ & MS Excel & $2010-2040$ \\
\hline PEN & Econométrico & Simulación & MS Excel & $2014-2025$ \\
\hline PLANCC & $\begin{array}{c}\text { Gas Natural: Uso Final } \\
\text { Resto: Econométrico }\end{array}$ & $\begin{array}{c}\text { Electricidad: Optimización } \\
\text { Resto: Simulación }\end{array}$ & MS Excel & $2012-2050$ \\
\hline SPEN & $\begin{array}{c}\text { Trasporte: Uso Final, Resto: } \\
\text { Econométrico }\end{array}$ & Optimización & TIMES & $2017-2040$ \\
\hline APEC & $\begin{array}{c}\text { Transporte: Uso Final } \\
\text { Residencia: Uso Final } \\
\text { Resto: Econométrico }\end{array}$ & Optimización & GAMS & $2016-2050$ \\
\hline
\end{tabular}

Tabla 3: Cumplimiento de paradigmas

\begin{tabular}{|c|c|c|c|c|c|}
\hline & $\begin{array}{c}\text { Objetivos } \\
\text { Cuantificados }\end{array}$ & $\begin{array}{c}\text { Evaluación de } \\
\text { Escenarios } \\
\text { Modelados }\end{array}$ & $\begin{array}{c}\text { Adaptarse a } \\
\text { complejidad } \\
\text { creciente }\end{array}$ & $\begin{array}{c}\text { Interacción con } \\
\text { otros sectores }\end{array}$ & $\begin{array}{c}\text { Transparencia, } \\
\text { reproductibilidad } \\
\text { e inteligibilidad }\end{array}$ \\
\hline EIEP & $S I$ & $N O$ & $N O$ & $N O$ & $N O$ \\
\hline NUMES & $S I$ & $\begin{array}{c}\text { Sl: Trade-Off y } \\
\text { Minimax }\end{array}$ & $N O$ & SI: Ambiental & $N O$ \\
\hline$P E N$ & $N O$ & $N O$ & $N O$ & $N O$ & $N O$ \\
\hline PLANCC & $S I$ & $N O$ & $N O$ & SI: Economía & $N O$ \\
\hline$S P E N$ & $N O$ & $N O$ & $N O$ & SI: Economía & $N O$ \\
\hline APEC & $S I$ & $N O$ & $N O$ & $N O$ & $N O$ \\
\hline
\end{tabular}

\subsection{Descripción del Modelo Propuesto}

A fin de cubrir la debilidad identificadas en los modelos empleados en los estudios analizados en la sección anterior, se ha estructurado una propuesta metodológica integral la cual se presenta en la figura 2, para ello se ha empleado una metodología híbrida que utiliza: i) una componente de uso final para la proyección de la demanda capaz de modelar los cambios en el parque de consumo energético, el efecto sustitución, la entrada de nuevas unidades y la obsolescencia de las mismas,
Asimismo, se podrá modelar los centros de transformación, cuantificar el nivel de reservas de energía primaria, consolidar los balances de energía y calcular las emisiones de GEl; ii) una componente de optimización para la expansión, operación y el cálculo de los costos marginales del sistema eléctrico, iii) una componente de integración de balances de energía y escenarios evaluados y iv) una componente de evaluación de escenarios simulados.

\section{A. Componente de uso final}


Un hito importante para el uso de esta metodología es la realización del estudio de balance de energía útil [31] realizada por el MINEM y que nos provee de información valiosa sobre el parque de consumo de energía. La componente de uso final utiliza el modelo LEAP [32] el cual nos permite analizar en detalle la demanda por uso final de energía por tipo de usuario, fuente y tecnología. Asimismo, analiza las diferencias estadísticas, los centros de transformación, las variaciones de stock y las reservas de recursos energéticos.

Debido al amplio grado de detalle que requiere este modelo, es necesario priorizar el análisis de los sectores en que los cambios tendenciales no reflejan apropiadamente lo que se espera para el largo plazo o los que se espera haya un impacto importante de las medidas de mitigación propuestas.

\section{B. Componente de optimización}

La componente de optimización utiliza el modelo PERSEO 2.0 [33] el cual nos permite optimizar el despacho de energía eléctrica a mínimo costo y determinar los costos marginales de energía en el SEIN. En primer lugar, realiza la lectura de los archivos de datos los cuales contienen toda la información relevante del sistema eléctrico nacional.

Seguidamente, efectúa la formulación matemática, para ello se han implementado en GAMS un conjunto de rutinas que traducen los datos ingresados en las ecuaciones matemáticas correspondientes. A continuación, se realiza la llamada a la herramienta de optimización que, en este caso, corresponde al optimizador CPLEX®. Este optimizador, se encarga de obtener la solución óptima de mínimo costo para cada una de las secuencias hidrológicas evaluadas, para ello dispone de varias técnicas de optimización lineal.

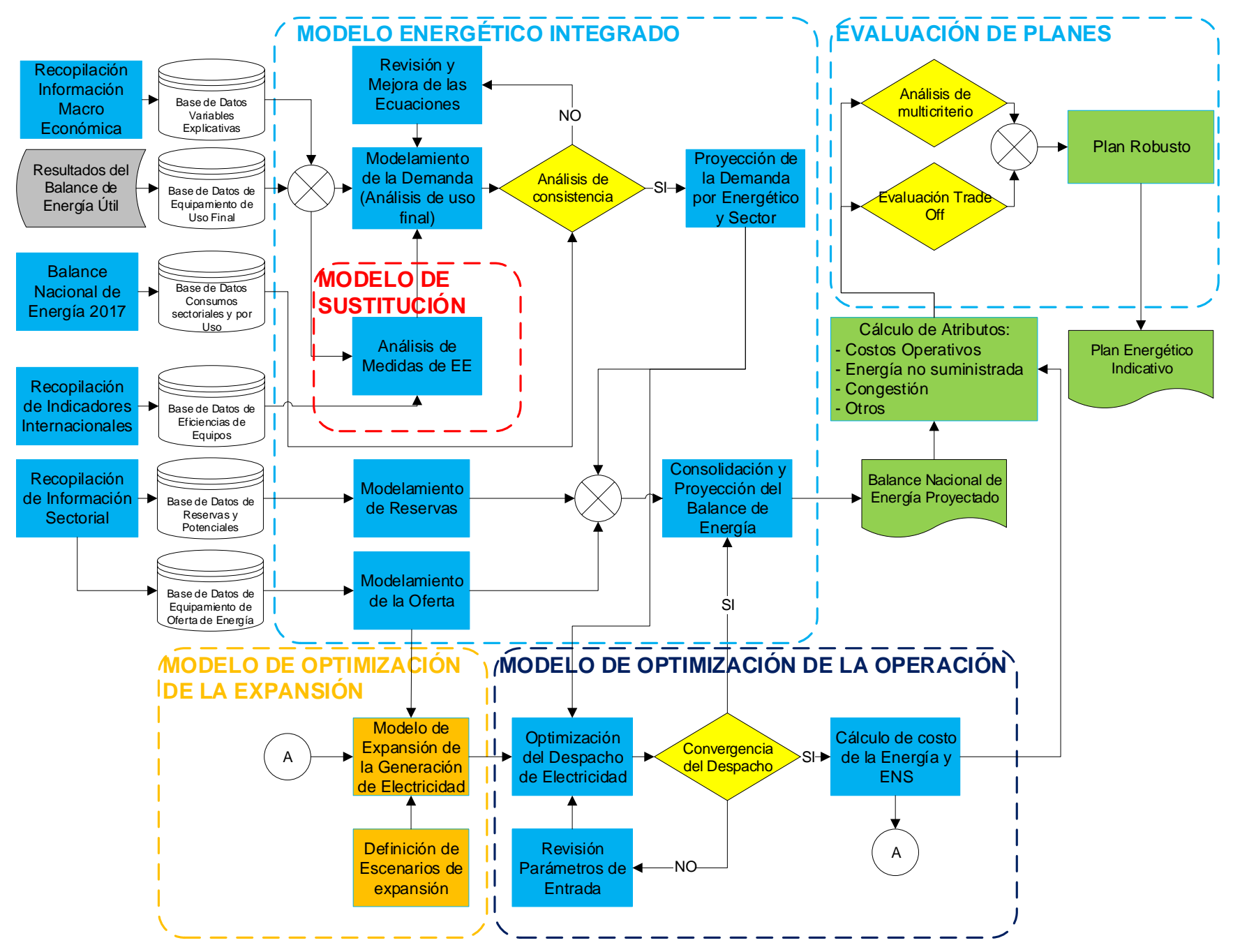

Figura 2: Propuesta Metodológica Integral 
Finalmente, se generan los reportes con un formato compatible con el MS EXCEL y son importados al LEAP para realizar la consolidación del balance nacional de energía.

\section{Consolidación y proyección de los balances de energía y cálculo de emisiones}

Sobre la base de los resultados de la proyección de la demanda de la componente de uso final y la proyección de la oferta de los centros de transformación (necesaria para cubrir la demanda) que incluye el despacho de electricidad resultante de la componente de optimización, se procede con la consolidación de los balances de energía.

El cálculo de emisiones se realiza sobre los resultados de los balances de energía, para ello, se hace uso de los factores de emisión del IPCC, aplicados en la Actualización del Inventario Nacional de Emisiones de Gases de Efecto Invernadero al Año 2009 [34]. [35].

\section{Componente de Evaluación de Escenarios Simulados}

Los escenarios simulados se evalúan para poder cuantificar los atributos de intereses de la política energética que pueden estar contrapuestos, el ejemplo típico son las emisiones de GEI contra la operación a mínimo costo del sistema, para ello, se puede emplear un análisis multicriterio o el análisis Trade-Off.

\subsection{Ventajas del modelo propuesto}

La metodología híbrida permite realizar un análisis por uso final de los energéticos donde se esperan cambios no tendenciales, como por ejemplo el notable incremento de la participación del consumo de gas natural en la matriz energética a partir de la puesta en marcha del proyecto Camisea [36].

El uso de una componente de optimización del despacho de electricidad nos permite determinar los costos marginales de la energía eléctrica con lo cual podemos calcular el impacto tarifario que tendrán los escenarios de medidas de mitigación evaluados.

El uso del LEAP permite consolidar los balances de energía y estimar de manera directa las emisiones de GEI y las reducciones esperadas por la implementación de las medidas de mitigación.

\subsection{Posibles dificultades}

La componente de uso final requiere de mucha información para la estructuración de la línea de base y es altamente dependiente del criterio de expertos para la estimación de tendencias y formulación de supuestos.

La componente de optimización requiere de mucha información referente a la composición del sistema eléctrico nacional, como las características técnicas de los grupos de generación actual y futuro, las barras del sistema donde se conectan, cuencas hidrográficas donde se ubican las centrales de generación y la cartera de proyectos futuros.

Asimismo, debió a que cuenta con 4 componentes donde se realizan cálculos por separado, se debe tener especial cuidado al momento de la integración de modelos.

\subsection{Oportunidades de Mejora}

Los resultados del modelo propuesto se pueden integrar a un modelo de equilibrio general de la economía, a fin de determinar los impactos de las medidas de mitigación en el desarrollo del sector económico. Los modelos de equilibrio general incorporan los shocks esperados para la economía en el periodo de análisis. Con ello se puede hacer un análisis de la compatibilidad entre los supuestos empleados para el sector energético (por ejemplo, tasas de crecimiento) y los que se obtengan del modelo de equilibrio para la economía.

Por otro lado, al tratarse de software abierto se cumple perfectamente con los paradigmas propuestos en la metodología ya que estará a disposición de la academia para futuros desarrollos y mejoras.

\section{Conclusiones}

En el Perú no existe un modelo energético integral que sea estandarizado y de software abierto que sirva de base para los estudios de prospectiva.

Los estudios de planeamiento realizados a la fecha han sido esfuerzos aislados que tuvieron metodologías marcadamente diferentes. Asimismo, no cumplen con la mayoría de los nuevos paradigmas que exigen los sistemas energéticos en la actualidad

La propuesta metodológica planteada busca cubrir el vacío de un sistema energético que pueda ser usado por todas las entidades del sector energético y de la academia. 


\section{Agradecimientos}

Agradecer a FONDECYT (Convenio $n{ }^{\circ}$ 207-2015FONDECYT) por su beca de doctorado en ciencias con mención en energética.

\section{Apéndice A Tabla de Acrónimos}

\begin{tabular}{|c|c|}
\hline APEC & $\begin{array}{l}\text { Asia Pacific Economic } \\
\text { Cooperation }\end{array}$ \\
\hline APERC & $\begin{array}{l}\text { Asia Pacific Energy Research } \\
\text { Centre }\end{array}$ \\
\hline BAU & Business as Usual \\
\hline CENERGIA & $\begin{array}{l}\text { Centro de Conservación de } \\
\text { Energía y del Ambiente }\end{array}$ \\
\hline CEPLAN & $\begin{array}{l}\text { Centro Nacional de Planeamiento } \\
\text { Estratégico }\end{array}$ \\
\hline COP & Conference of parties \\
\hline DGEE & $\begin{array}{l}\text { Dirección General de Eficiencia } \\
\text { Energética }\end{array}$ \\
\hline GAMS & $\begin{array}{l}\text { General Algebraic } \quad \text { Modeling } \\
\text { System }\end{array}$ \\
\hline GEI & Gases de Efecto Invernadero \\
\hline IEEJ & $\begin{array}{l}\text { The Institute of Energy Economic, } \\
\text { Japan }\end{array}$ \\
\hline iNDC & $\begin{array}{l}\text { Intended Nationally Determined } \\
\text { Contributions }\end{array}$ \\
\hline IPCC & $\begin{array}{lll}\text { Intergovernmental Panel on } \\
\text { Climate Change }\end{array}$ \\
\hline LEAP & $\begin{array}{l}\text { Long-range Energy Alternatives } \\
\text { Planning }\end{array}$ \\
\hline MAPS & $\begin{array}{llll}\text { Mitigation } & \text { Action } & \text { Plans } & \text { and } \\
\text { Scenarios } & & & \\
\end{array}$ \\
\hline MEF & $\begin{array}{llll}\text { Ministerio de } & \text { Economía y } \\
\text { Finanzas }\end{array}$ \\
\hline MINAM & Ministerio del Ambiente \\
\hline MINEM & Ministerio de Energía y Minas \\
\hline $\begin{array}{l}\text { PERSEO } \\
2.0\end{array}$ & $\begin{array}{l}\text { Modelo para la optimización del } \\
\text { despacho hidrotérmico en los } \\
\text { procesos regulatorios }\end{array}$ \\
\hline PLANCC & $\begin{array}{l}\text { Planificación ante el Cambio } \\
\text { Climático }\end{array}$ \\
\hline PROSEMER & $\begin{array}{l}\text { Programa para la Gestión } \\
\text { Eficiente y Sostenible de los } \\
\text { Recursos Energéticos del Perú }\end{array}$ \\
\hline RREE & $\begin{array}{l}\text { Ministerio de Relaciones } \\
\text { Exteriores }\end{array}$ \\
\hline SEIN & $\begin{array}{l}\text { Sistema Eléctrico Interconectado } \\
\text { Nacional }\end{array}$ \\
\hline TGT & Escenario de Metas propuestas \\
\hline TIMES & $\begin{array}{l}\text { The Integrated MARKAL-EFOM } \\
\text { System }\end{array}$ \\
\hline $2 \mathrm{DC}$ & $\begin{array}{l}\text { Escenario de incremento máximo } \\
\text { de } 2^{\circ} \text { centígrados }\end{array}$ \\
\hline
\end{tabular}

\section{Referencias}

[1] Organización Latinoamericana de Energía Manual de Planificación Energética, 2nd ed. (OLADE, Quito, Ecuador, 2017), pp. 212.

[2] Pfenninger S, Hawkes A y Keirstead J, Renewable and Sustainable Energy Reviews 33 (2014) 74-86.

[3] Fragkos $P$ y Kouvaritakis N, Energy 160 (2018) 965-978.

[4] Nieto J, Carpintero O y Miguel L, Ecological Economics 146 (2018) 69-84.

[5] Postic S, Selosse S y Maizi N, Energy Policy 101 (2017) 170-184.

[6] Ministerio del Ambiente, Contribución prevista y determinada a nivel nacional (iNDC) de la república del Perú, (Ministerio de Ambiente, Perú, 2015).

[7] Ministerio de Energía y Minas, DS N0642010-EM, (Diario oficial el peruano, Perú, 24/11/2010).

[8] Gerencia Regulación Tarifaria, Fijación de Precios en Barra Periodo mayo 2019 - abril 2020 (OSINERGMIN, Perú, 2019).

[9] Comité de Operación Económica del Sistema Interconectado Nacional, Actualización del Plan de Transmisión 2021-2020 (COES, Perú, 2019).

[10] Ministerio de Energía y Minas, Plan de Referencial de Electricidad 2008-2017, (Ministerio de Energía y Minas, Perú, 2008).

[11] Gerencia Regulación Tarifaria, Procedimiento para aprobación del Plan de Inversiones en Transmisión, Período 20212025 (OSINERGMIN, Perú, 2019).

[12] Ministerio de Energía y Minas, Norma Criterios y Metodología de Planificación para la Elaboración del Plan de Inversión en Distribución Eléctrica (Pre publicado) (MINEM, Perú, 2018).

[13] Dirección General de Electrificación Rural, Plan Nacional de Electrificación Rural 2016 - 2025 (MINEM, Perú, 2015).

[14] Dirección General de Hidrocarburos, Plan de Desarrollo de los Recursos de Hidrocarburos 2017 - 2021 (MINEM, Perú, 2018).

[15] Gerencia de Regulación de Tarifas División de Gas Natural, Plan Quinquenal de Inversiones 2018-2022 (OSINERGMIN, Perú, 2018).

[16] N. Van Beeck, Classification of Energy Models, (FEW Research Memorandum, 
Tilburg University, The Netherlands, 1999, pp. 17-23.

[17] Urban F, Benders R y Moll H, Energy Policy 35 (2007) 3473-3482.

[18] Hall L, Buckley A, Applied Energy 169 (2016) 607-628.

[19] Huppmann D, Gidden M, Fricko O, Kolp P, Orthofer C, Environmental Modelling and Software 112 (2019) 143-156

[20] Oficina Técnica de Energía, Estudio Integral Energético de Perú, (Ministerio de Energía y Minas, Perú, 2001)

[21] Dirección General de Eficiencia Energética, Nueva Matriz Energética Sostenible y Evaluación Ambiental Estratégica, como Instrumentos de Planificación, (Ministerio de Energía y Minas, Perú, 2012)

[22] J.E. Luyo, Urge un plan energético consensuado para el desarrollo sostenible del Perú, (Grupo de Estudos do Setor Elétrico UFRJ, TDSE 49, Rio de Janeiro, 2012, pp. 3-23.

[23] Dirección General de Eficiencia Energética, Plan Energético Nacional 2014 - 2025, (Ministerio de Energía y Minas, Perú, 2014)

[24] A. Ríos, Futuro de la Energía en el Perú: Estrategias Energéticas Sostenibles, (International Rivers, Berkeley, USA, 2016, pp. 146-156.

[25] Proyecto Planificación ante el Cambio Climático, Escenarios de Mitigación del Cambio Climático en el Perú al 2050: Construyendo un Desarrollo Bajo en Emisiones, (PLANCC, Perú, 2014)

[26] Cohen B, Tyler E y Torres M, Climate Policy 17 (2017) 1065-1075.
[27] Chávez J, Development 59 (2016) 342-349.

[28] Programa para la gestión eficiente y sostenible de recursos energéticos del Perú, Presentación Sistema de Planificación Energética, (Ministerio de Energía y Minas, 2019).

[29] Asia Pacific Economic Cooperation, APEC Energy Demand and Supply Outlook, 7th ed. - Volume II (Asia Pacific Energy Research Centre and The Institute of Energy Economics Japan, Tokyo, 2019) pp. 273-292.

[30] Asia Pacific Economic Cooperation, APEC Energy Demand and Supply Outlook, 7th ed. - Annex I: Modelling key assumptions \& methodologies (Asia Pacific Energy Research Centre and The Institute of Energy Economics Japan, Tokyo, 2019).

[31] Dirección General de Eficiencia Energética, Balance de Energía Útil 2013, (Ministerio de Energía y Minas, Perú, 2017)

[32] Heaps C.G., Long-range Energy Alternatives Planning (LEAP) system, [Software version 2018.1.32] (Stockholm Environment Institute, Somerville, MA, USA, 2016)

[33] Gerencia de Regulación Tarifaria, Modelo PERSEO 2.0, (OSINERGMIN, Perú, 2018)

[34] Ministerio del Ambiente, Inventario Nacional de Gases de Efecto Invernadero 2012, (Ministerio de Ambiente, Perú, 2016).

[35] Grupo Intergubernamental de Expertos sobre Cambio Climático, Guidelines for National Greenhouse Gas Inventories, (IGES, Japón, 2006)

[36] Oficina de Estudios Económicos, ¿Qué significa el Proyecto Camisea?, (OSINERGMIN, Perú, 2006)

E-mails: jnmeza@gmail.com jeluyo@yahoo.es 